\title{
Determinants of Age at Menarche Studied in Adolescents Residing in Guwahati, India
}

\author{
Dr. Varsha C. R., $\mathrm{MD}^{1}$, Dr.Gayatri Bezboruah, $\mathrm{MD}^{2}$, \\ ${ }^{1}$ consultant Pediatrician, Shirur Hospital, Bagalkot, Karnataka \\ ${ }^{2}$ Prof And HOD Of Pediatrics, Gauhati Medical College, Guwahati, Assam
}

\begin{abstract}
Menarche is a vital event of puberty in female adolescents. The aim of this study was To determine the average age of menarche in the urban population of Guwahati, Assam and to assess the role of body composition, socioeconomic status, diet, mother's age at menarche etc as determinants of age at menarche. This observational study was conducted for one year at government recognized schools in Guwahati city. 500 school going girls aged between 9 to 15 years who have attained menarche were included. Data collection was done by questionnaires, personal interviews and physical examination.

Results: The average age at menarche in this study was found to be 12.32+/-0.982 years. There exists a statistically significant positive correlation between mother and daughters' age at menarche. There is no statistically significant correlation between age at menarche and the body mass index of the child .There was statistically significant correlation between age at menarche and socioeconomic status, menarche occurring earlier in higher socioeconomic classes. On review of available literature, it was found that there is a secular trend towards a decline in the age at menarche in Assam at the rate of 3.94 months per decade.
\end{abstract}

\section{Introduction}

Menarche is a vital event of puberty in female adolescents. Menarche is a distinct event with a sudden onset. It is the most accurately recalled indicator of puberty among girls. During the past century, there has been a secular (time-related) trend towards an earlier onset of menarche in most developed countries. This decline in menarcheal age is expected in Indian girls considering the economic development of the country in the past few decades

\section{Aims And Objectives}

(1) To determine the average age of menarche in the urban population of Guwahati, Assam, India.

(2) To assess the role of body composition, socioeconomic status, family size, mother's age at menarche etc as determinants of age at menarche

(3) To determine if there is a secular trend towards decline of age at menarche.

\section{Materials And Methods}

a) Study design: Analytical (cross sectional study)

b) Setting: Government recognized schools in Guwahati city, Kamrup district, Assam.

c) Study period: A period of 1 year from 1st August 2011 to 31st July, 2012

d) Study population: Girls aged between 9 years to 15 years

e) Inclusion criteria:

1. Girl child aged between nine to sixteen years

2. Attending selected Government recognized schools in Guwahati city, Kamrup District, Assam and residing in Guwahati city

3. The study subjects should have attained menarche i.e. should have had their first menstrual period.

4. Parents of the subject should be able to provide required information through pretested Performa or personal interview.

f) Exclusion criteria:

1. Girls who have not attained menarche

2. Children whose parents/guardians do not give consent for participation

h) Sample size:

500 school going girls of ages between 9 to 15 years.

i) No randomization/blinding/intervention was done in the study. 


\section{k) Data collection methods}

Each school authority was approached and the intention of the study was explained.

Informed consent was taken from the principal of the school.

Data collection was done by questionnaires, personal interviews and physical examination

- All subjects between the ages of 9-15 yrs were interviewed to elicit details regarding their age, class, any chronic illness in the past, previous hospitalization, if the child is on any long-term treatment, age of attainment of menarche

- The data pertaining to mothers and family was collected through a self-administered pretested questionnaire sent through the respective children

- Data regarding occupation of parents, family size, type of housing, monthly income was collected and the subjects assigned into different socioeconomic strata according to modified Kuppuswamy classification.

- Examination was done in the school premises under the supervision of a senior staff.

- Weight and height were obtained, BMI was calculated by the formula weight $(\mathrm{kg}) /\{\text { height }(\mathrm{mt})\}^{2}$ and each child was categorized according to percentiles based on the 2007 WHO reference standards.

- The SMR staging was done as per Tanner's staging with the cooperation of the child

\section{l) Data management and statistical analysis}

The data thus collected was subsequently scrutinized individually and analyzed Proportions were compared using "Chi square "or "Fisher exact test". Repeated measures over time were evaluated using ANOVA for repeated measures. A Probability of 5\% was considered significant. Data were entered into Epi 2000 software and analyzed using both Epi 2000 and SPSS analytical software.

\section{Results and observations}

Age at menarche in the study population

Table 1:

\begin{tabular}{|c|c|c|}
\hline Age at menarche & Frequency & Percent \\
\hline 9 & 2 & 0.4 \\
\hline 10 & 18 & 3.6 \\
\hline 11 & 75 & 15.0 \\
\hline 12 & 169 & 33.8 \\
\hline 13 & 199 & 39.8 \\
\hline 14 & 33 & 6.6 \\
\hline 15 & 4 & 0.8 \\
\hline Total & 500 & 100.0 \\
\hline
\end{tabular}

Table 1 shows the age at menarche of the study population. $75(15 \%)$ attained menarche at 11 years of age, $169(33.8 \%)$ at 12 years of age, $199(39.8 \%)$ at 13 years of age, $33(6.6 \%)$ attained at 14 years of age and 4 $(0.8 \%)$ attained menarche at 15 years of age. The average age at menarche in this study was found to be 12.32+/-0.982 years

Duration of menstrual bleeding 5.65 days

In this study the average number of days of menstrual bleeding in the study subjects was found to be

Duration of menstrual cycle

The study showed that $72 \%$ had cycle duration of $\mathbf{2 1}-\mathbf{3 5}$ days i.e. within the normal range. $11.2 \%$ girls were found to have cycle duration of $<21$ days and $16.2 \%$ had cycle duration of $>35$ days

Month wise onset of Menarche 
Fig 1:

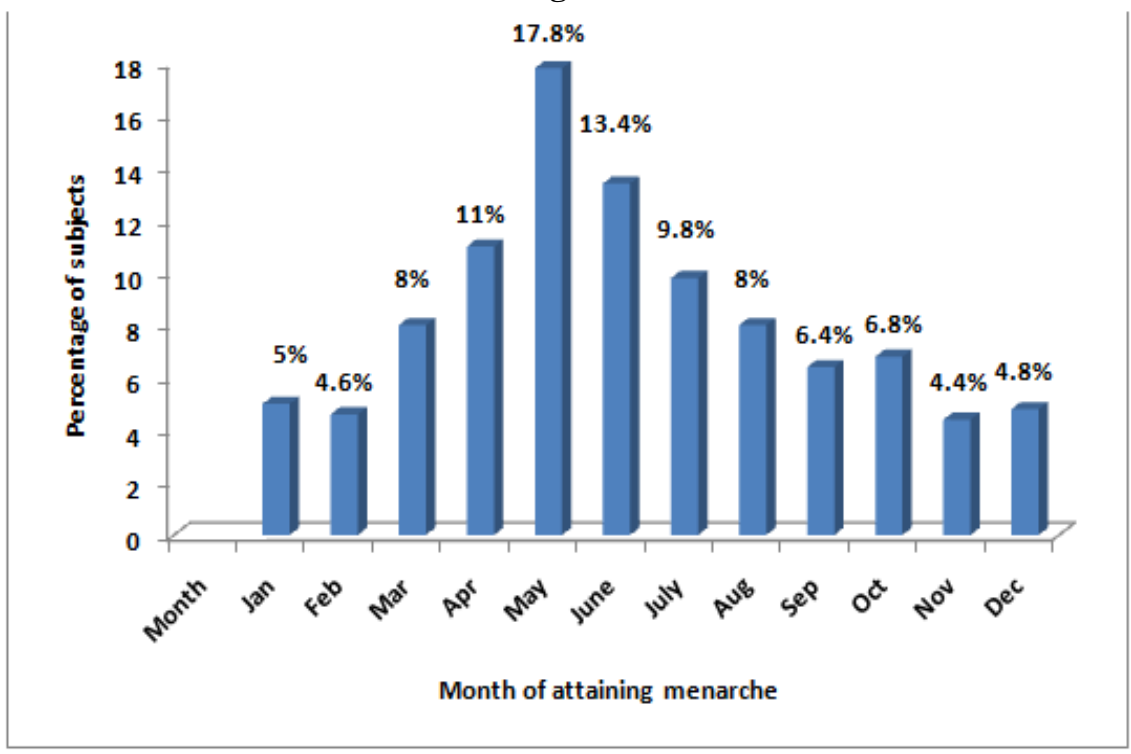

It was found in this study that the most common months of attainment menarche were the summer months and least common were winter months and the values were statistically significant. ( $p$ value 0.041 ).

\section{Age at menarche of Mothers of the study subjects}

Fig 2:

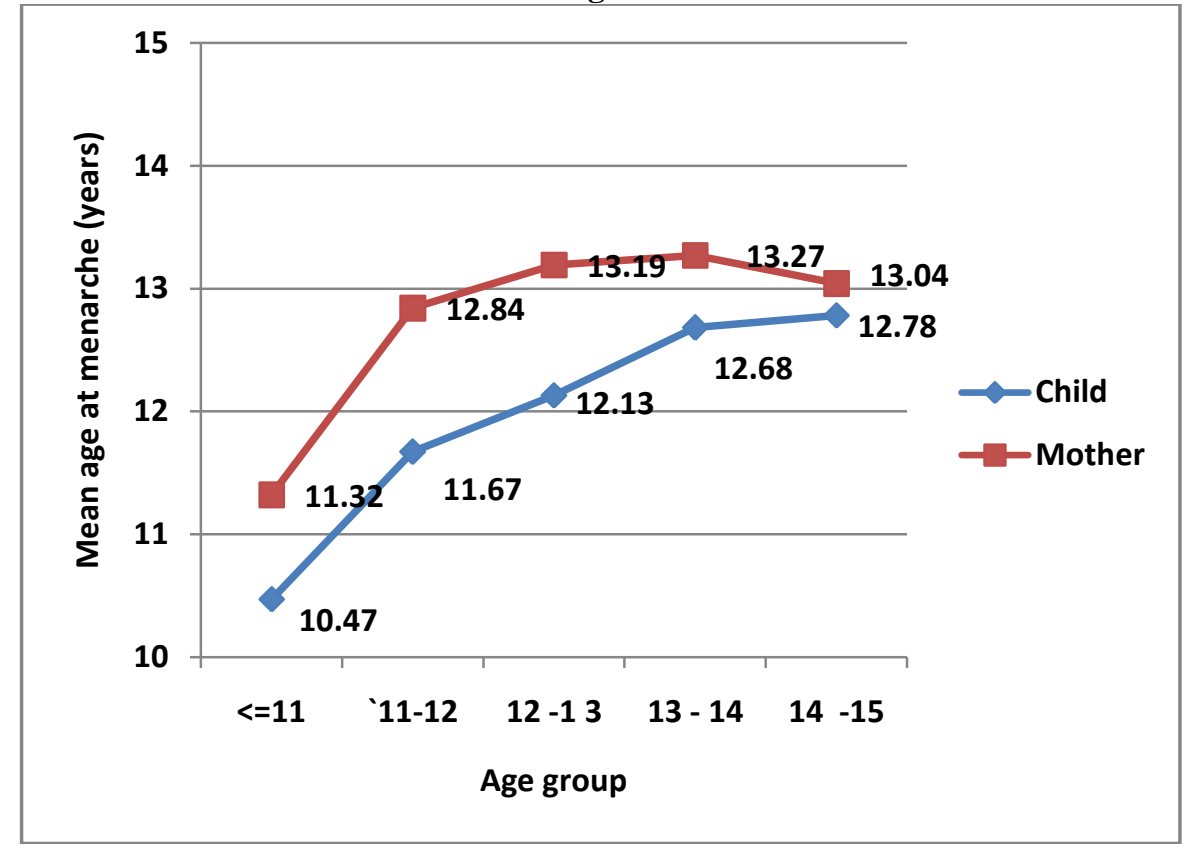

Figure 2 shows that there exists a statistically significant positive correlation between mother and daughters' age at menarche.

\section{Correlation between Age at menarche and BMI}

\begin{tabular}{|} 
Table 2: \\
\begin{tabular}{|c|c|c|}
\hline & R & p-value \\
\hline $\begin{array}{c}\text { Correlation of age at menarche and } \\
\text { BMI }\end{array}$ & 0.036 & 0.423 \\
\hline
\end{tabular}
\end{tabular}

Table 2 shows that there is no statistically significant correlation between age at menarche and the body mass index of the child. 
Determinants Of Age At Menarche Studied In Adolescents Residing In Guwahati, India

Age at menarche and socioeconomic status (classified according to Kuppuswamy classification)

Table 3:

\begin{tabular}{|c|c|c|c|c|c|}
\hline Socioeconomic class & $\mathbf{N}$ & $\begin{array}{c}\text { Mean age at menarche } \\
\text { (years) }\end{array}$ & Std. Deviation & Minimum & Maximum \\
\hline Upper & 30 & 11.47 & .973 & 9 & 13 \\
\hline Upper middle & 290 & 12.31 & .901 & 10 & 15 \\
\hline Lower middle & 102 & 12.47 & .982 & 10 & 14 \\
\hline Upper lower & 52 & 12.48 & 1.093 & 10 & 15 \\
\hline Lower & 25 & 12.48 & 1.194 & 10 & 15 \\
\hline
\end{tabular}

Table 3 shows that the subjects belonging to upper, upper middle, lower middle, upper lower and lower socioeconomic classes attained menarche at 11.7, 12.31, 12.47, 12.48 and 12.48 years respectively. These values were found to be statistically significant ( $\mathrm{p}$ value $<0.001)$.

\section{Age at menarche and Family size}

Table 4:

\begin{tabular}{|c|c|c|c|c|c|}
\hline Family size & $\mathbf{N}$ & Mean & Std. Deviation & Minimum & Maximum \\
\hline$<=3$ & 104 & 12.60 & .940 & 10 & 15 \\
\hline $\mathbf{4 . 0 0}$ & 280 & 12.26 & 1.009 & 9 & 15 \\
\hline $\mathbf{5 . 0 0}$ & 50 & 12.24 & .981 & 10 & 14 \\
\hline$>=6$ & 66 & 12.20 & .863 & 10 & 14 \\
\hline
\end{tabular}

Table 4 shows that the average age at menarche was higher in girls with smaller family size and comparatively lower in girls belonging to larger families. These values were found to be statistically significant ( $p$ value $0.014)$.

\section{Discussion}

Guwahati is called the Gateway to the north-east, a fast growing educational, industrial and economic hub. This makes the study on the age and determinants of menarche the need of the hour.

\section{Secular trend in the age at menarche}

The lowering of age at menarche at an average rate to 3-4 months in some countries of Europe, North America, and several parts of the world has been well documented. ${ }^{1,2,3}$ It reflects the improved socio-economic, nutritional and general health conditions in these countries.

In 1953 Baruah found the mean age at menarche in Assam to be 14.29 years. In 1954, Rakshid studied pooled sample of Assamese girls and found mean age of menarche to be 13.39 years ${ }^{4}$

Sengupta S, Gogoi G, Chetry H B in 1996 studied 571 girls in Dibrugarh, Assam and found the mean age at menarche to be $12.23+/-0.19$ years $^{5}$

Deb R in 2009 studied 453 girls from Guwahati, Assam and found the mean age at menarche to be 12.45+/-0.02 years. ${ }^{6}$

In the present study mean age at menarche was found to be $12.32+/-0.982$ years.

Thus this study shows that there is a secular trend towards a decline in the age at menarche in Assam at the rate of 3.94 months per decade. Also, the daughters' ages at menarche have been found consistently lesser than their mothers' ages at menarche. This may further support the theory of secular trend towards decline in age at menarche

The average number of days of menstrual bleeding was found to be 5.65 days in this study. Bhalla M, Srivastava J R, 1974, reported that majority of the girls tend to have their menarcheal age around the same age as their mothers.

Towne r, Czerwinski S, Demerath E et al in 2005, drew conclusions from the Fels longitudinal study and found that the Heritability of age of menarche was $0.49+/-0.13 .{ }^{7,8}$

Many studies (Acharya V, P.Reddaiah, N, Baridalyne, 2006, Currie C, Ahluwalia N, Godeau E, 2010) in the past have suggested an inverse correlation between nutritional status and age at menarche i.e. menarche appears earlier in girls with better nutritional status. ${ }^{9,10}$

But in this study the correlation between BMI and age at menarche were not found to be statistically significant. Further prospective studies are required to assess the relation of BMI and age at menarche as this study considers the post menarcheal BMI which may not always reflect the BMI before attainment of menarche. On performing ANOVA it was found that the difference in age at menarche between different socioeconomic classes was statistically significant i.e. girls belonging to higher socio economic status attained menarche earlier compared to girls belonging to lower socioeconomic status. 
Bagga A.; Kulkarni S and Kahl H, Schaffrath Rosario A, Schlaud M also showed an inverse correlation between age at menarche and socioeconomic status. ${ }^{11,12}$

This study showed that the average age at menarche was higher in girls with smaller family size and comparatively lower in girls belonging to larger families. These values were found to be statistically significant. Deb R, 2006 in a study conducted in Guwahati found that menarche occurred earlier in girls belonging to smaller families similar to the present study. ${ }^{6}$

\section{Conclusion}

Menarche no doubt is the most important event during female puberty. This study showed that the mean age at menarche in Guwahati city, Assam is $12.32+/-0.982$ years. This finding is comparable to studies conducted in India and abroad in the recent years.

This study has shown evidence that there is a definite secular trend towards decrease in age at menarche at the rate of 3.94 months per decade. It thus seems that the age at onset of menarche is governed by a complex interaction of environmental and genetic factors. The secular trend is a reflection of change in the social and environmental scenario of the country. Further longitudinal studies are warranted to understand the effect of earlier onset of menarche.

\section{Competing interests: none}

Funding: none

\section{References}

[1]. Khanna G,Kapoor S; Secular Trend in Stature and Age at Menarche Among Punjabi Aroras Residing in New Delhi, India; Coll. Antropol. 28 (2004) 2: 571-575,UDC 572.087:618.172

[2]. Harris MA, Prior JC, Koehoorn M; Age at menarche in the Canadian population: secular trends and relationship to adulthood BMI; J Adolesc Health. 2008 Dec;43(6):548-54.

[3]. Dewhurst j;"Female puberty abnormalities" series ed, Singer A and Jordan J., Edinburgh,Churchill Livingstone,1984,204pp

[4]. Agarwal A N,Agarwal D K, Upadhyay S K; Physical and sexual growth pattern of affluent Indian children from 8 to 15 years of age; Indian pediatrics, vol29, oct 92,1204-1282

[5]. Sengupta S, Gogoi G, Chetry HB Variation in menarcheal age of Assamese girls.J Indian Med Assoc. 1996 Mar;94(3):88-90

[6]. Deb R.; Variation in the Age at Menarche of the Assamese and Bengali girls of Guwahati, Assam, Anthropologist, 11(4): 259-264 (2009)

[7]. $\quad$ Singh G.M.P.”Onset of menarche in girls of Ludhiana” Indian Pediatrics ,1974;11(2);99-105

[8]. Towne r,Czerwinski S, Demerath E;Heritability of age of menarchefrom the Fels longitudinal study; American j. of physical anthropometry; 128:210-219(2005)

[9]. Acharya A, Reddaiah V.P ,Nutritional Status and Menarche in Adolescent Girls in an Urban Resettlement Colony of South Delhi, Indian Journal of Community Medicine Vol. 31, No. 4,302-303, October-December, 2006

[10]. Currie C,Ahluwalia N, Godeau E; Is Obesity at Individual and National Level Associated With Lower Age at Menarche? Evidence From 34 Countries in the Health Behaviour in School-aged Children Stu Journal of Adolescent Health 50 (2012) 621-626

[11]. Bagga A.;KulkarniS Age at menarche and secular trend in Maharashtrian (Indian) girls Volume 44(1-4):53-57, 2000 Acta Biologica Szegediensis

[12]. Kahl H, Schaffrath Rosario A, Schlaud M.; Sexual maturation of children and adolescents in Germany,Results of the German Health Interview and Examination Survey for Children and Adolescents Bundesgesundheitsblatt Gesundheitsforschung Gesundheitsschutz. 2007 May-Jun;50(5-6):677-85 